\title{
Cigarette smoking and schizophrenia
}

\author{
Ciara Kelly \& Robin McCreadie
}

It has been recognised for many years that patients with psychiatric illnesses, especially schizophrenia, smoke to excess. However, recently there has been a resurgence of interest in the high prevalence of nicotine addiction in patients with schizophrenia. It has become increasingly clear that heavy cigarette smoking is intimately associated with the schizophrenic illness and that this may have implications for the underlying neurobiology of schizophrenia.

\section{Rates of cigarette smoking}

The number of patients with schizophrenia who smoke is very high (see Box 1 ). One study reported the prevalence to be $88 \%$, nearly three times the rate in the general population and higher than the elevated rates of smoking in patients with other psychiatric illnesses. The increased prevalence persists even after adjustment for marital status, alcohol use and socio-economic status (Hughes et al, 1986).

A number of more recent cross-sectional studies from different countries have reported high rates of smoking in patients with schizophrenia (Goff et al, 1992; Chong \& Choo, 1996). One large study of 360 state hospital in-patients, of whom 237 were diagnosed as having schizophrenia or schizoaffective disorder, found that the overall prevalence of smoking was $85 \%$, and $93 \%$ in young male patients with schizophrenia (De Leon et al, 1995). Smoking occurs at much higher rates than other types of substance misuse or dependence, which have been shown also to be elevated among patients with schizophrenia.
In our own study (Kelly \& McCreadie, 1999) we found that the average age when patients with schizophrenia started smoking was the same as in the general population, namely mid-teens; $90 \%$ of patients who smoked had started smoking before their illness began.

Patients with schizophrenia who smoke are also heavier smokers than those in the general population and those with other psychiatric disorders. In our own study, $68 \%$ of patients with schizophrenia who smoked were classed as heavy smokers ( 25 or more cigarettes daily) compared with only $11 \%$ of the general population who smoke. In another study (Olincy et al, 1997), patients with schizophrenia who smoked had much higher levels of the nicotine metabolite cotinine in comparison with other smokers, confirming our finding. Excessive smoking tends to be a lifelong habit among patients with schizophrenia. The proportion of those who quit is lower than in the general population; in our study of a population of patients with schizophrenia, only $8 \%$ of males were ex-smokers, compared with $31 \%$ of males in a local general population.

\section{Box 1 Rates of smoking in schizophrenia}

The rate of smoking in people with schizophrenia is at least two to three times that in the general population

Patients who smoke, smoke at heavier rates than in the general population

Most patients start smoking in their teens, before the illness begins

Ciara Kelly is a lecturer in the Department of Psychological Medicine at Glasgow University (Academic Department, Gartnavel Royal Hospital, 1055 Great Western Road, Glasgow, G12 0XH). Her current research interests are the lifestyle of patients with schizophrenia and their cognitive function. Robin McCreadie is Director of Clinical Research at Crichton Royal Hospital, Dumfries. He has published widely on the Nithsdale cohort of patients with schizophrenia. 


\section{Why do patients with schizophrenia smoke?}

Why do patients with schizophrenia smoke at these excessive rates? There are three possible explanations for the association: something about the illness leads patients to smoke; smoking is another risk factor for schizophrenia; or a third factor leads to both schizophrenia and smoking (see Box 2).

The first possibility has received most attention. It has been suggested that smoking may be a marker of a more severe illness process (De Leon, 1996). Smokers are more often young and male; they have an earlier onset of illness, increased numbers of hospital admissions and receive higher doses of neuroleptic medication (Goff et al, 1992; Ziedonis et al, 1994). In addition, smokers have more severe symptoms with higher scores on the Brief Psychiatric Rating Scale (Overall \& Gorham, 1962) for positive and negative symptoms (Goff et al, 1992; Chong \& Choo, 1996; Ziedonis et al, 1994).

Another suggestion is that patients smoke as a form of self-medication with nicotine, which may help regulate a dysfunctional mesolimbic dopamine system. It may increase dopamine release in the pre-frontal cortex and alleviate positive and negative symptoms (Lavin et al, 1996). Worsening of psychotic symptoms on nicotine withdrawal has been reported (Dalack \& Meador-Woodruff, 1996). It has also been shown that nicotine administration enhances cognitive performance on a number of tasks. However, in general, patients with schizophrenia who smoke report similar reasons to other smokers ("addicted", "relaxation" and "to calm down"), with only $17 \%$ of patients reporting that smoking improved psychotic symptoms.

As we have found that most patients who smoke began to do so before psychotic aspects of the illness appeared, premorbid characteristics are perhaps important. It is noteworthy that in our study patients who smoked were as children more poorly adjusted socially than those who were not smokers.

Box 2 Why do patients with schizophrenia smoke?

Aspects of the illness might lead more patients to smoke

Smoking might be an aetiological factor in schizophrenia

Genetic and/or environmental factors might lead both to nicotine addiction and to schizophrenia
Patients with schizophrenia may smoke heavily as a result of antipsychotic medication, which produces marked dopamine receptor blockade. Possibly, a very high level of smoking is necessary to overcome this blockade and produce the reward effects. It has been shown that, compared with baseline, patients with schizophrenia smoke more after starting haloperidol (McEvoy et al, 1995). It has also been shown in a placebo-controlled study that after a single dose of haloperidol, normal subjects smoked significantly more during the following hour, as measured by blood nicotine levels (Dawe et al, 1995).

A second explanation for the association between schizophrenia and smoking is that smoking acts as an aetiological risk factor for schizophrenia. It may be that repeated activation by nicotine of the mesolimbic system over a long time precipitates the onset of schizophrenia in vulnerable individuals. We found that the earlier the age of starting smoking, the earlier was the onset of psychotic illness in women (Kelly \& McCreadie, 1999). Interestingly, nicotine acts like other drugs of addiction such as cocaine and amphetamine, activating the mesolimbic dopamine system (Pontieri et al, 1996); this effect appears to be of critical importance for the reinforcing and reward properties of the drug. Also, nicotine has been shown to increase burst activity in the dopamine neurons of the ventral tegmental area, a form of firing pattern of these cells that is physiologically associated with basic motivational processes underlying learning and cognition (Iversen, 1996).

Third, genetic and/or environmental factors might predispose individuals to develop both schizophrenia and nicotine addiction. Much work in the genetics of both schizophrenia (Maier \& Schwab, 1998) and nicotine addiction (Clarke, 1998) has focused on the dopamine receptor system.

\section{Consequences of smoking}

\section{Morbidity and mortality}

Smoking remains the single greatest preventable cause of death in our society. It appears to engender both general and specific health risks for patients with schizophrenia. There is no doubt that cigarette smoking causes considerable morbidity and mortality, but there are no epidemiological data addressing smoking-related morbidity and mortality in schizophrenia. Previous work has shown that among people with schizophrenia life expectancy is estimated as being $20 \%$ less than in the general population. There is an increase in deaths from 
natural causes and the most common causes are cardiovascular and respiratory disease, both smoking-related (Mortensen \& Juel, 1993). The markedly elevated prevalence of smoking is clearly an important potential factor in explaining the elevated mortality in schizophrenia.

\section{Medication and side-effects}

Smoking has been shown to decrease plasma levels of neuroleptics by inducing hepatic microsomal enzymes (Salokangas et al, 1997). Therefore, patients who smoke require larger doses of drugs than nonsmokers to achieve the same therapeutic effect (Lohr \& Flynn, 1992). It has also been reported that smokers have been prescribed neuroleptics at twice the daily dose of non-smokers while exhibiting significantly less neuroleptic-induced parkinsonism. Spontaneous abnormal involuntary movements indistinguishable from tardive dyskinesia have also been reported in a general population sample of older males who smoke (Nilsson et al, 1997). It would seem likely, therefore, that there is an association between tardive dyskinesia and smoking in schizophrenia. However, in three previous studies, only one showed that tardive dyskinesia was more prevalent among smokers (Yassa et al, 1987); two found no relationship (Menza et al, 1991; Chiles et al, 1993).

\section{Economic considerations}

Cigarettes are not cheap. We have recently carried out some simple arithmetic in relation to smoking and schizophrenia (McCreadie \& Kelly, 2000). Our patients smoke on average 26 cigarettes per day. At $£ 2.79$ per packet, this works out at $£ 1300$ per year. Very few of our patients work. We reckon that probably up to one-third of state benefits is spent on cigarettes. Of the cost of a packet of cigarettes, $87 \%$ is tax. Thus, patients, through their smoking habits, are contributing very substantially to the cost of their own care.

\section{Smoking cessation}

Smoking cessation rates are poor in heavy smokers and even lower in those with psychiatric illness. Attempts to get patients with schizophrenia to stop smoking have met with only limited success. There is often a perception that it will be unsuccessful and will deprive individuals of one of their few pleasures. This view is inherently discriminatory. In our own study (Kelly \& McCreadie, 1999) a third of patients reported that they wanted to quit for health reasons.
Box 3 Management issues

Smoking must be implicated in the increased mortality in schizophrenia

Smokers require higher doses of antipsychotic medication

A substantial proportion of the income of smokers with schizophrenia is spent on cigarettes

Patients with schizophrenia have the right to be offered treatment for their nicotine addiction

Clearly, smokers with schizophrenia have a severe nicotine addiction, and pharmacological and psychological support with smoking cessation needs to be addressed.

There have been few studies that have looked at cessation methods (reviewed by Lavin et al, 1996). Smoking bans on in-patient psychiatric units have generated naturalistic reports; usually the most severely addicted patients are extremely resourceful and continue to smoke. Smoking cessation groups, nicotine gum, nicotine patches and clonidine patches have all been tried with both in-patients and outpatients, with poor results. For example, the sixmonth cessation rate was only 12-13\% with group therapy alone (Addington et al, 1998) or in combination with nicotine reduction therapy (Ziedonis \& George, 1997). Outcome studies are urgently necessary to evaluate the effectiveness of smoking cessation programmes (e.g. in-patient $v$. out-patient programmes, nicotine replacement, behavioural techniques and so on). For the severely addicted, the following combinations may be tried: combined gum and patch therapy; bupropion and nicotine patch; nicotine nasal spray; or nicotine inhaler (details available from the author upon request).

The use of bupropion is interesting. In normal subjects, sustained-release bupropion, an antidepressant, alone or in combination with a nicotine patch, resulted in significantly higher long-term rates of smoking cessation (30.3 and $35.5 \%$, respectively) than use of either the nicotine patch alone $(16.4 \%)$ or placebo $(15.6 \%)$ (Jorenby et al, 1999). In a recent case report (Evins \& Tisdale, 1999), sustainedrelease bupropion was successfully used with a chronically ill male patient with schizophrenia. Why might an antidepressant such as bupropion be helpful in smoking cessation? It has been suggested that long-term administration of bupropion and nortriptyline, adrenergic antidepressants, produces a significantly increased responsivity to stimulation of the ventral tegmental dopamine cells. This is not seen with selective serotonin reuptake 
inhibitors (SSRIs). This may be why buproprion and nortriptyline but not SSRIs increase smoking abstinence. For a fuller comment on this possibility, see Glassman (1998).

We have recently reported (Scottish Schizophrenia Research Group, 2000) that the prevalence of smoking in first-episode patients is just as high as in chronic patients, but that the number of cigarettes smoked per day is no higher than that in smokers in the general population. Perhaps it is at this early stage that attempts to help patients to stop smoking should be made.

In conclusion, despite the often limited resources of patients with schizophrenia, they commonly view cigarette smoking as a necessity. Most patients with schizophrenia smoke at high rates and few manage to stop. This dramatically elevated prevalence of smoking among individuals with schizophrenia raises the intriguing possibility that nicotine use and schizophrenia have some shared underlying neurobiology.

\section{References}

Addington, J., el-Guebaly, N., Campbell, W., et al (1998) Smoking cessation treatment for patients with schizophrenia. American Journal of Psychiatry, 155, 974-976.

Chiles, J. A., Cohen, S., Roland, M., et al (1993) Smoking and schizophrenic psychopathology. American Journal of Addictions, 2, 315-319.

Chong, S. A. \& Choo, H. L. (1996) Smoking among Chinese patients with schizophrenia. Australian and New Zealand Journal of Psychiatry, 30, 350-353.

Clarke, P. B. S. (1998) Tobacco smoking, genes and dopamine. Lancet, 353, 84-85.

Dalack, G. W. \& Meador-Woodruff, J. H. (1996) Smoking, smoking withdrawal and schizophrenia: case reports and a review of the literature. Schizophrenia Research, 22, 133141.

Dawe, S., Gerada, C., Russell, M. A., et al (1995) Nicotine intake in smokers increases following a single dose of haloperidol. Psychopharmacology, 117, 110-115.

De Leon, J. (1996) Smoking and vulnerability for schizophrenia. Schizophrenia Bulletin, 22, 405-409.

- Dadvand, M., Canuso, C., et al (1995) Schizophrenia and smoking: an epidemiological survey in a state hospital. American Journal of Psychiatry, 152, 453-455.

Evins, A. E. \& Tisdale, T. (1999) Bupropion and smoking cessation. American Journal of Psychiatry, 156, 798-799.

Glassman, A. H. (1998) Psychiatry and cigarettes. Archives of General Psychiatry, 55, 692-693.

Goff, D. C., Henderson, D .C. \& Amico, E. (1992) Cigarette smoking in schizophrenia: relationship to psychopathology and medication side-effects. American Journal of Psychiatry, 149, 1189-1194.

Hughes, J. R., Hatsukami, D. K., Mitchell, J. E., et al (1986) Prevalence of smoking among psychiatric outpatients. American Journal of Psychiatry, 143, 993-997.

Iversen, L. L. (1996) Smoking ... harmful to the brain. Nature, $382,206-207$.

Jorenby, D. E., Leischow, S. J., Nides, M. A., et al (1999) A controlled trial of sustained release bupropion, a nicotine patch, or both for smoking cessation. New England Journal of Medicine, 304, 685-691.

Kelly, C. \& McCreadie, R. G. (1999) Smoking habits, current symptoms, and premorbid characteristics of schizophrenic patients in Nithsdale, Scotland. American Journal of Psychiatry, 156, 1751-1757.
Lavin, M. R., Siris, S. G. \& Mason, S. E. (1996) What is the clinical importance of cigarette smoking in schizophrenia? American Journal of Addictions, 5, 189-208.

Lohr, J. B. \& Flynn, K. (1992) Smoking and schizophrenia. Schizophrenia Research, 8, 93-102.

McCreadie, R. G. \& Kelly, C. (2000) Patients with schizophrenia who smoke. Private disaster, public resource. British Journal of Psychiatry, 176, 109.

McEvoy, J. P., Freudenreich, O., Levin, E. D., et al (1995) Haloperidol increases smoking in patients with schizophrenia. Psychopharmacology, 119, 124-126.

Maier, W. \& Schwab, S. (1998) Molecular genetics of schizophrenia. Current Opinion in Psychiatry, 11, 19-25.

Menza, M. A., Grossman, N., Van Horn, M., et al (1991) Smoking and movement disorders in psychiatric patients. Biological Psychiatry, 30, 109-115.

Mortensen, P. B. \& Juel, K. (1993) Mortality and causes of death in first admitted schizophrenic patients. British Journal of Psychiatry, 163, 183-189.

Nilsson, A., Waller, L., Rosengren, A., et al (1997) Cigarette smoking is associated with abnormal involuntary movements in the general male population. Biological Psychiatry, 41, 717-723.

Olincy, A., Young, D. A. \& Freedman, R. (1997) Increased levels of the nicotine metabolite cotinine in schizophrenic smokers compared to other smokers. Biological Psychiatry, $42,1-5$.

Overall, J. E. \& Gorham, D. R. (1962) The brief psychiatric rating scale. Psychological Reports, 10, 799-812.

Pontieri, F. E., Tanda, G., Orzi, F., et al (1996) Effects of nicotine on the nucleus accumbens and similarity to those of addictive drugs. Nature, 382, 255-256.

Salokangas, R. K. R., Saarijarvi, S., Taiminen, T., et al (1997) Effect of smoking on neuroleptics in schizophrenia. Schizophrenia Research, 23, 55-60.

Scottish Schizophrenia Research Group (2000) Smoking habits and plasma lipid peroxide and vitamin E levels in nevertreated first-episode patients with schizophrenia. Preliminary report. British Journal of Psychiatry, 176, 290-293.

Yassa, R., Lal, S., Korpassy, A., et al (1987) Nicotine exposure and tardive dyskinesia. Biological Psychiatry, 22, 67-72.

Ziedonis, D. M., Kosten, T. R., Glazer, W. M., et al (1994) Nicotine dependence and schizophrenia. Hospital and Community Psychiatry, 45, 204-206.

- \& George, T. P. (1997) Schizophrenia and nicotine use report of a pilot smoking cessation programme and review of neurobiological and clinical issues. Schizophrenia Bulletin 23, 247-254.

\section{Multiple choice questions}

1. Patients with schizophrenia who smoke:

a smoke at rates higher than those with other psychiatric conditions

$\mathrm{b}$ in the early stages of the illness smoke more cigarettes daily than general population smokers

c start smoking at a later age than general population smokers

d quit at the same rates as general population smokers.

2. Nicotine may:

a regulate a dysfunctional mesolimbic dopamine system

b improve psychiatric symptoms on withdrawal

c impair cognitive performance

d prevent relapse. 
3. Smoking cessation in schizophrenia:

a can be achieved more readily than in other psychiatric conditions

$b$ is unwanted by more than two-thirds of patients themselves

c should probably be attempted at the early stages of the illness

$\mathrm{d}$ might include combined nicotine gum and patch therapy.

\begin{tabular}{|c|c|c|}
\hline 1 & 2 & 3 \\
\hline a $T$ & a $\mathrm{T}$ & $\mathbf{a}$ \\
\hline b F & b F & b \\
\hline c $F$ & c $\quad F$ & c \\
\hline d F & d F & d \\
\hline
\end{tabular}

$F$

b

d $\mathbf{T}$

\section{The Royal College of Psychiatrists Forthcoming Conferences}

Faculty of Child and Adolescent Psychiatry Residential

18-20 September 2000

Kensington Town Hall, London

Faculty for the Psychiatry of Learning Disability Annual Residential Meeting

4-6 October 2000

Jurys Hotel, Cork, Ireland

The Older Driver with Mental Health Problems: Clinical, Legal and Ethical Issues 12 October 2000

Society for Chemical Industry, 15 Belgrave Square, London

Philiosophy Special Interest Group of the Royal College of Psychiatrists The Second R.D. Laing Conference

21 October 2000

The School of Oriental and African Studies, London

Residential Meeting of the Section of Rehabilitation and Social Psychiatry

9-10 November 2000

Swansea Marriott Hotel

ECT Training Day

7 December 2000

King's Fund, London (to attend this training day please contact Alex Cellini tel: 0131220 2910)

For more information please contact the Conference Office, The Royal College of Psychiatrists, 17 Belgrave Square, London SW1X 8PG.

Tel: +44 (0)20 72352351 ext 142; Fax: +44 (0)20 72451231 